\title{
Analysis of a Compact Modulator Incorporating a Hybrid Silicon/Electro-Optic Polymer Waveguide
}

\author{
Kjersti K. McLauchlan, Student Member, IEEE, and Scott T. Dunham, Senior Member, IEEE
}

\begin{abstract}
The design and simulation of a novel resonant cavity optical modulator is presented in this paper. The device incorporates a hybrid silicon/electro-optic (EO) polymer slot waveguide in the cavity region with distributed Bragg reflectors in single-mode silicon waveguide regions at each end of the cavity to create a modulator with a large modulation depth, short device length, and a low drive voltage for use in future high-speed integrated optics applications. Full three-dimensional simulations of this device structure are computationally very expensive; therefore, the periodicity of the device has been exploited to allow for a cascade matrix approach to be employed to reduce the time and computational resources necessary for accurate simulation of light propagation during the optimization phase of the device design.
\end{abstract}

Index Terms-Integrated optics, optical modulation, silicon photonics.

\section{INTRODUCTION}

$\mathbf{M}$ ANY research groups have demonstrated the use of silicon or electro-optic (EO) polymers in Mach-Zehnder (MZ) modulator devices. However, the MZ modulators are generally quite long, and it is challenging to achieve high-speed optical devices in silicon [1]. Also, because the refractive indexes of polymers are generally much lower than that of silicon and therefore closer to the refractive index of the cladding materials, polymer waveguides must generally have much larger dimensions than those of silicon guides for good modal confinement. Recent advances in EO polymer design have resulted in fastresponse polymers with large EO coefficients $(\sim 100 \mathrm{pm} / \mathrm{V})$, which allow for high-speed switching with lower applied voltages [2]. A common figure of merit for the MZ modulators is the $V_{\pi} L$ product, which combines the length of the active region and applied voltage necessary to achieve a phase change of $\pi$ adians at the output. The current state-of-the-art high-speed silicon MZ modulator utilizes a MOS capacitor structure developed by Liu et al. and exhibits a $V_{\pi} L$ product of $\sim 8 \mathrm{~V}-\mathrm{cm}$ with a $1 \mathrm{GHz}$ modulation bandwidth [1]. An MZ modulator fabricated using a highly nonlinear chromophore (CLD) and an amorphous polycarbonate (APC) in a guest-host EO polymer exhibited a $V_{\pi} L$ product of $2.2 \mathrm{~V}-\mathrm{cm}$ and a bandwidth of 110 $\mathrm{GHz}$ [3]. The standard material for MZ modulators is currently lithium niobate, which has excellent stability and low optical

Manuscript received October 14, 2005; revised August 9, 2006. This paper was supported in part by a National Science Foundation Graduate Research Fellowship and in part by the National Science Foundation Center on Materials and Devices for Information Technology Research (CMDITR) under Grant DMR-0120967.

The authors are with the Electrical Engineering Department, University of Washington, Seattle, WA 98195-2500 USA (e-mail: kkleven@ee. washington.edu; dunham@ee.washington.edu).

Digital Object Identifier 10.1109/JSTQE.2006.884090

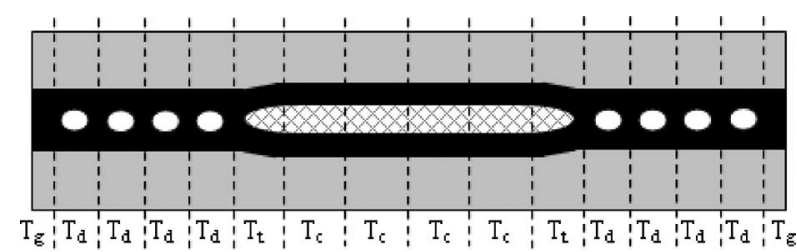

Fig. 1. Top view of the resonant cavity modulator. Silicon (black). Silicon dioxide of an SOI structure (gray). Air (white). EO polymer in the hybrid waveguide structure (gray cross-hatch area). Sections used for the cascade matrix simulation are also shown.

loss, but has a $V_{\pi} L$ product of approximately $4.5 \mathrm{~V}-\mathrm{cm}$ with push-pull electrodes [4].

Resonant cavity devices, such as Fabry-Perot or microring resonators, can generally have smaller device dimensions, but it is challenging to achieve large modulation depths required for the optical communication applications. Barrios et al. fabricated and tested a silicon Fabry-Perot resonant cavity modulator with a total device length of $19 \mu \mathrm{m}$ [5], [6]. This modulator used freecarrier injection and thermal effects to achieve the phase change in the resonant cavity region. It required an applied voltage of $3.7 \mathrm{~V}$ to achieve a modulation depth of $53 \%$. A microring resonant device fabricated with the CLD/APC polymer had a ring radius of $750 \mu \mathrm{m}$, and the voltage necessary to shift the resonant peak by the full-width at half-maximum (FWHM) was $16 \mathrm{~V}$ [7].

The structure proposed here combines the benefits of the high index of refraction and manufacturability of silicon with the strong, high-speed response of the EO polymer in a hybrid waveguide resonant cavity modulator to create a high-speed device with a low drive voltage, large modulation depth, and compact size. The analysis uses an alternative modeling approach to significantly reduce the computational resources necessary to perform three-dimensional (3-D) analysis, which is critical for the device design and optimization.

\section{Hybrid Waveguide Structure}

This paper looks specifically at the resonant cavity modulator design incorporating a hybrid silicon/EO polymer slot waveguide in the cavity region with distributed Bragg reflectors in the silicon waveguide (shown in Figs. 1 and 2). Almeida et al. have described a waveguide in which light can be guided through a void nanostructure if there are narrow high-index ridges on each side of the void region [8]. The intensity of the light is enhanced in the void region due to the boundary conditions enforced by Maxwell's equations at the interfaces. If a low-index EO polymer is used instead of a void region, the field will be similar to that of the void/silicon slot waveguide. However, this alternative structure also allows a field to be applied across the EO 


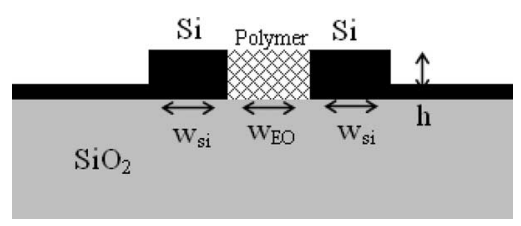

Fig. 2. Cross section of hybrid waveguide structure in the cavity region illustrating the design parameters.

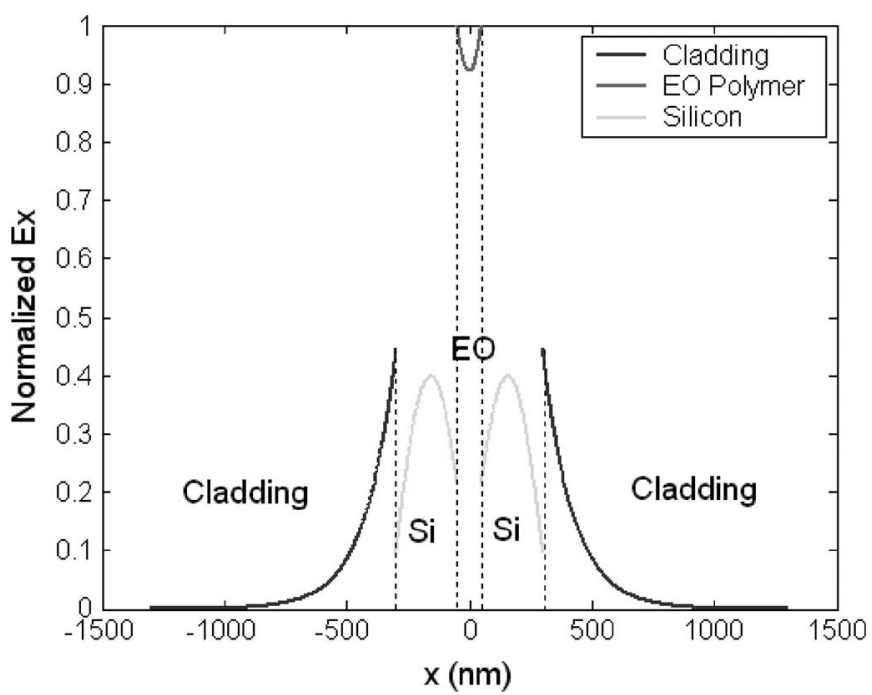

Fig. 3. Field profile for a $100 \mathrm{~nm}$ polymer core with $250 \mathrm{~nm}$ silicon ridges as calculated with an effective index approach.

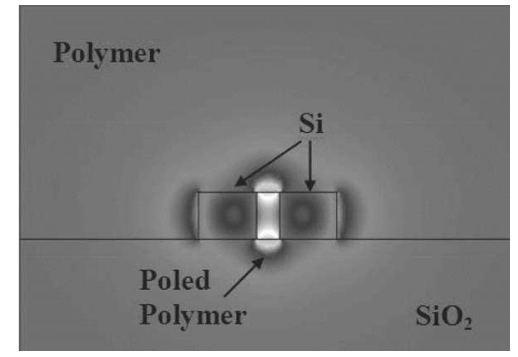

Fig. 4. Cross section of the field profile from a finite element simulation for a $100 \mathrm{~nm}$ polymer core with $250 \mathrm{~nm}$ silicon ridges showing enhanced field intensity in the core.

polymer region to cause a change in the propagation constant of the silicon/polymer waveguide, thereby modulating the output intensity at a given wavelength in the resonant cavity modulator.

The confinement of light in the low-index region is generally not what would be expected, as light is normally confined to the higher index material. However, when the dimensions of the high-index material are such that they do not allow for individual guided modes, the solution to Maxwell's equations results in a hybrid mode. The differences in the indexes of refraction between silicon and EO polymer result in a field that is highly confined to the polymer region in the hybrid waveguide, as illustrated in Figs. 3 and 4.

The dimensions of the hybrid waveguide were optimized to achieve the largest change in the propagation constant for a given

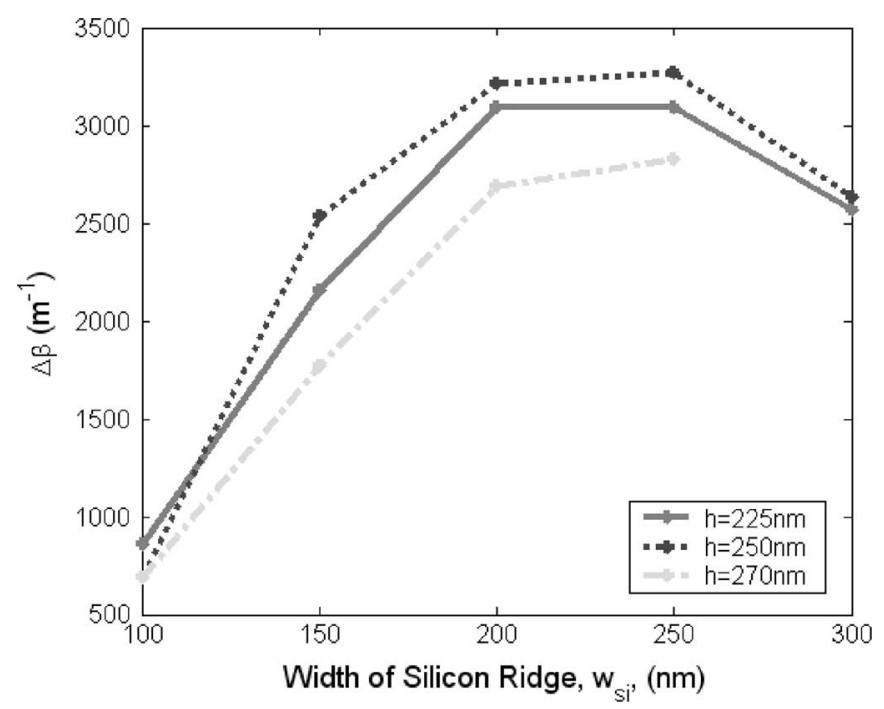

Fig. 5. Plot of propagation constant versus ridge width for various heights with a constant polymer gap spacing $(100 \mathrm{~nm})$ and applied voltage shows that there are optimum dimensions of the waveguide to achieve the greatest change in the propagation constant.

change in the index of refraction of the EO polymer. We consider AJL8 as the chromophore incorporated at $25 \%$ weight in APC, which has an index of refraction of 1.6 and an approximate EO coefficient, $r_{33}$, of $100 \mathrm{pm} / \mathrm{V}$ at $1.55 \mu \mathrm{m}$ [2], [9]. Therefore, a change in the propagation constant of the bulk polymer for a typical $2 \mu \mathrm{m}$ electrode spacing and $1 \mathrm{~V}$ appliedvoltage would be $\sim 400 \mathrm{~m}^{-1}$, as given by

$$
\Delta \beta=\beta_{1}-\beta_{2}=\frac{2 \pi}{\lambda_{0}}\left(n_{1}-n_{2}\right)
$$

where $\lambda_{0}$ is the wavelength in free space, $n_{1}$ is the index of refraction with no applied voltage, and $n_{2}$ is the index of refraction with an applied voltage $V_{a}$, as given by

$$
n_{2}=n_{1}-\frac{n_{1}^{3} r_{33} V_{\mathrm{a}}}{2 w_{\mathrm{eo}}}
$$

Note that if the polymers were used in a waveguide structure, this change in the propagation constant would be even less as some of the modes would be in the substrate and cladding regions. For reference, the change in the propagation constant of a bulk EO polymer with the same electric field as in the hybrid structure would be $8300 \mathrm{~m}^{-1}$; however, achieving this electrode spacing would lead to high losses in a traditional modulator structure with external electrodes.

The parameters of the hybrid waveguide that can be varied include the height and width of the silicon ridges as well as the spacing between the two ridges. The choice of the waveguide height will be an important decision, as the waveguide should be as large as possible to improve the coupling efficiency. However, as Fig. 5 shows, there are optimum dimensions for the hybrid waveguide to achieve the greatest change in the propagation constant. As the figure illustrates, wider and taller silicon ridges are beneficial until they become large enough to strongly support a guided mode. Once this limit is reached, there is no longer a 


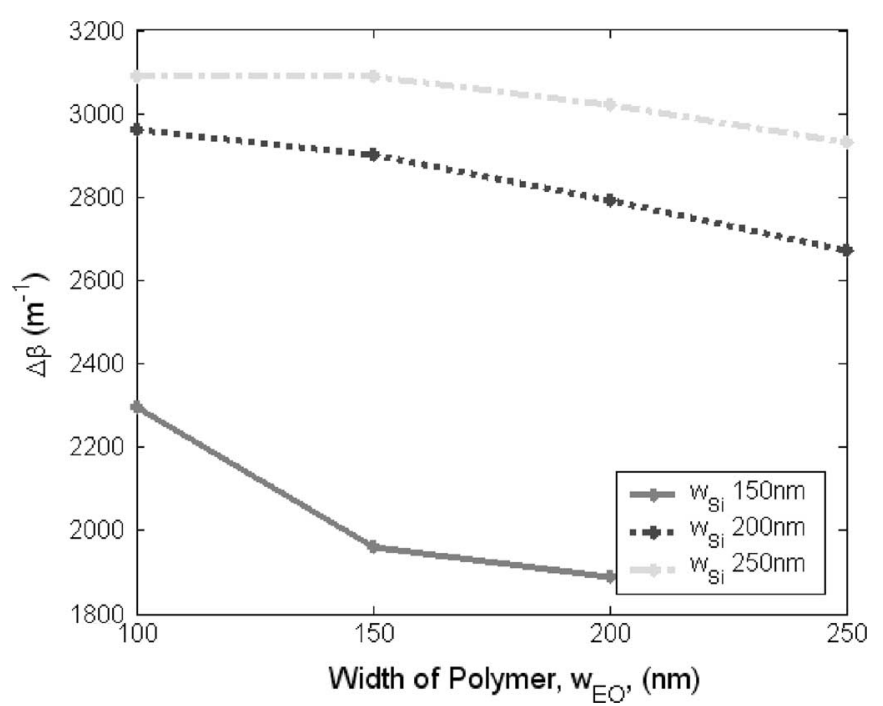

Fig. 6. Change in the propagation constant versus polymer gap width for a change of -0.003 in the index of refraction of the EO polymer in the core of the hybrid waveguide.

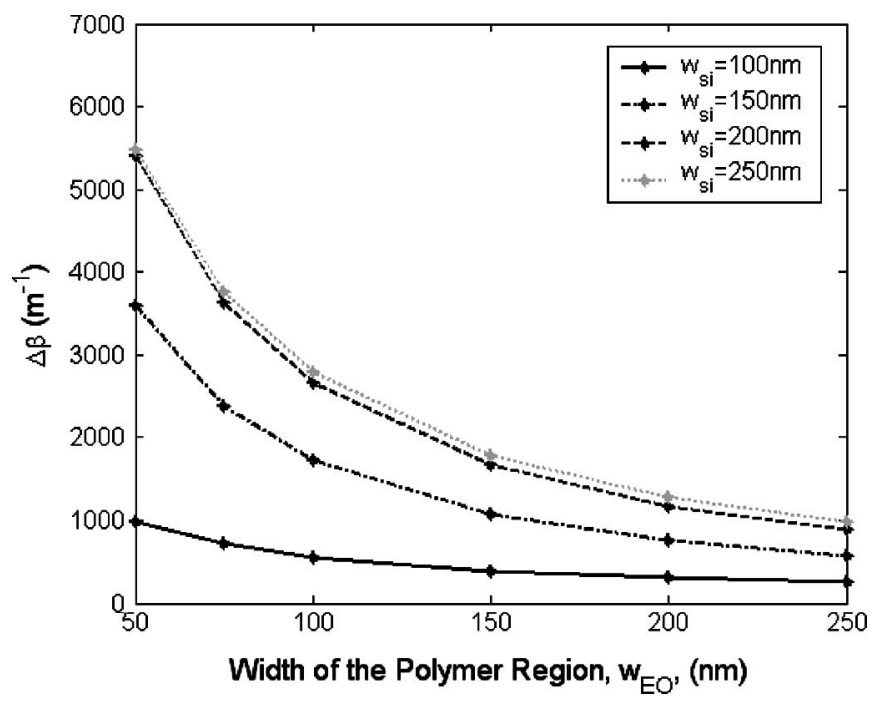

Fig. 7. Change in the propagation constant versus gap width using the AJL8 polymer $\left(n_{1}=1.6, r_{33}=100 \mathrm{pm} / \mathrm{V}\right)$ with $V_{a}=1 \mathrm{~V}$.

hybrid waveguide structure, but rather two separately guided modes in the silicon ridges.

Fig. 6 shows the achievable change in the propagation constant for the hybrid structure with a change in the index of refraction of the EO polymer of -0.003 as determined through finite element simulations. Some modulator applications may require a particular applied voltage, rather than a desired change in the index of refraction. With this in mind, the dimensions of the hybrid waveguide that would allow for the greatest change in the propagation constant with $V_{a}=1 \mathrm{~V}$ were also considered, and the results are shown in Fig. 7. With the hybrid structure, the silicon ridges can also be used as electrodes, which significantly reduces the electrode spacing and therefore increases the change in the index of refraction for a given applied voltage. Also illustrated in these figures are the optimal hybrid

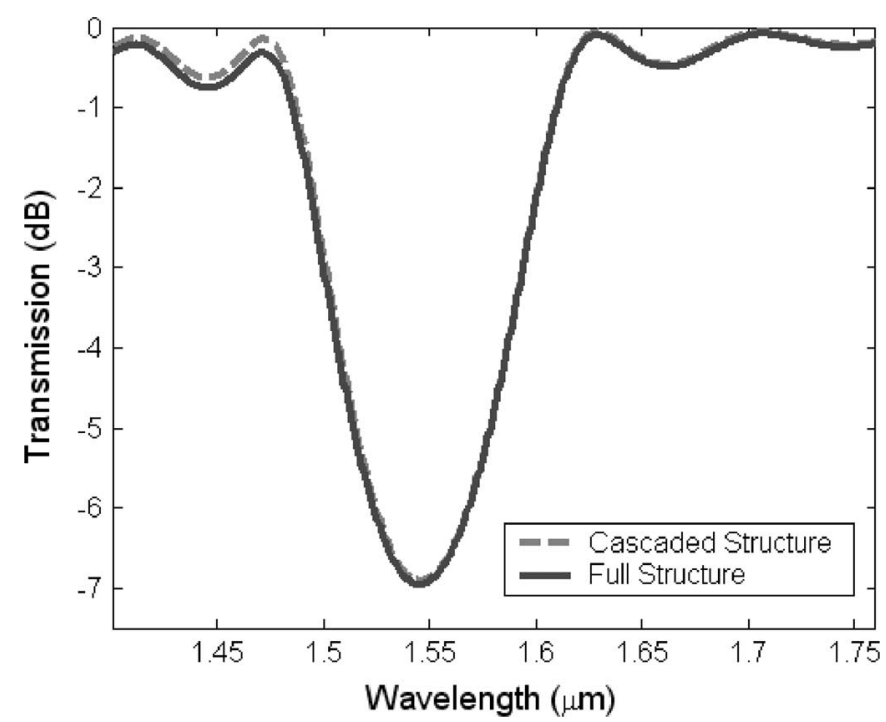

Fig. 8. Comparison of simulations for a seven-period PBG illustrating the agreement between the full simulation and the cascaded structure in the region of device operation.

waveguide parameters for achieving a large change in the propagation constant. Following this analysis, a hybrid waveguide with $250 \mathrm{~nm}$ square silicon ridges and $100 \mathrm{~nm}$ EO polymer core was chosen for the subsequent modulator design since these parameters would allow for a large-scale fabrication with current lithography techniques while simultaneously achieving a very large change in the propagation constant. The achievable index change of $2800 \mathrm{~m}^{-1}$ is substantially greater than that of an allpolymer waveguide with typical electrode spacing $\left(\sim 400 \mathrm{~m}^{-1}\right)$ and about one third of that for the bulk polymer with the same field $\left(8300 \mathrm{~m}^{-1}\right)$.

\section{DeVice ANALYSIS AND Simulation}

Due to the complex nature of the structure, high operating frequencies, and small device dimensions, 3-D simulations are required to obtain accurate propagation characteristics, particularly scattering losses. However, full-wave simulation of this device is computationally very expensive, especially for design optimization. This paper takes advantage of the periodicity of the resonant cavity structure by utilizing cascade matrixes to reduce simulation times [10]. With this approach, only the small unique sections of the device need to be simulated separately with 3-D full-wave modeling. The entire device structure can then be modeled by using matrix multiplication of the transmission characteristics $(\boldsymbol{T})$ of individual sections in the appropriate order (Fig. 1)

$$
\vec{T}=\vec{T}_{g} \vec{T}_{d} \vec{T}_{d} \vec{T}_{d} \vec{T}_{d} \vec{T}_{t} \vec{T}_{c} \vec{T}_{c} \vec{T}_{c} \vec{T}_{c} \vec{T}_{t} \vec{T}_{d} \vec{T}_{d} \vec{T}_{d} \vec{T}_{d} \vec{T}_{g}
$$

A comparison of the simulation of a seven-hole periodic bandgap structure with $100 \mathrm{~nm}$ holes is shown in Fig. 8 and demonstrates excellent agreement between the full structure simulation and the cascade matrix approach. The simulation time for the full seven-period structure was $10.2 \mathrm{~h}$, while a single-period simulation took less than $25 \mathrm{~min}$ to complete, 
which is a significant decrease in the analysis time. The projected simulation time for the full 3-D analysis of the entire structure, even with a relatively short cavity length, would be more than a month, while the cascade approach required less than $1 \mathrm{~h}$ for all of the individual sections.

Reduction in the computation time for this device configuration is even more significant because the entire device can be analyzed and optimized with only a small set of simulations, and the effect of parameters such as the number of periods, cavity length, and hole spacing can be analyzed without performing additional simulations. It is also possible to easily incorporate design changes using this method; a change in the hole shape, type of cavity structure, or hole size requires only that section be simulated again rather than the whole device.

\section{RESULTS}

The resonant cavity structure allows for design tradeoffs among the modulation depth, given in (4), the maximum transmission at the resonant wavelength, the FWHM, which is the width of the response at half of the maximum transmission, necessary applied voltage, and the length of the device

$$
M=\frac{I_{\max }-I_{\min }}{I_{\max }}
$$

In general, a longer cavity will result in a decrease in the FWHM and an increase in the modulation depth for a given number of periodic reflectors. However, future integrated optics applications are expected to require short device lengths along with a narrow FWHM and a large modulation depth. Therefore, variations in the number and size of the periods need to be considered in conjunction with the cavity length and the necessary applied voltage to determine the appropriate design choices for a given application.

The following analysis uses a change in the index of refraction of the EO polymer of -0.003 in the cavity region. The resulting modulation depths with 100-nm holes and various cavity lengths are shown in Fig. 9. From this figure, it can be seen that a cavity length of $15 \lambda$ is required to achieve a modulation depth greater than $90 \%$; however, as the number of reflectors is increased, the maximum transmission rapidly falls off to levels that may not be acceptable for detection purposes. Therefore, one must consider both the modulation depth and the maximum transmission when choosing the device characteristics.

Increasing the number of periods in the Bragg reflector increases the modulation depth and decreases the FWHM up to a saturation point. However, each additional hole included in the system adds scattering loss and increases the device length, and a larger hole diameter increases the amount of loss per period. This increase in loss significantly reduces the maximum transmission that can be achieved for a given cavity length and limits the achievable modulation depth. Therefore, for a given hole size, there is a limit of the number of additional periods that improve the performance. Once this limit is reached, the losses increase much more rapidly than does the reflectivity and dominate the response; this effect is clearly illustrated in Fig. 10.

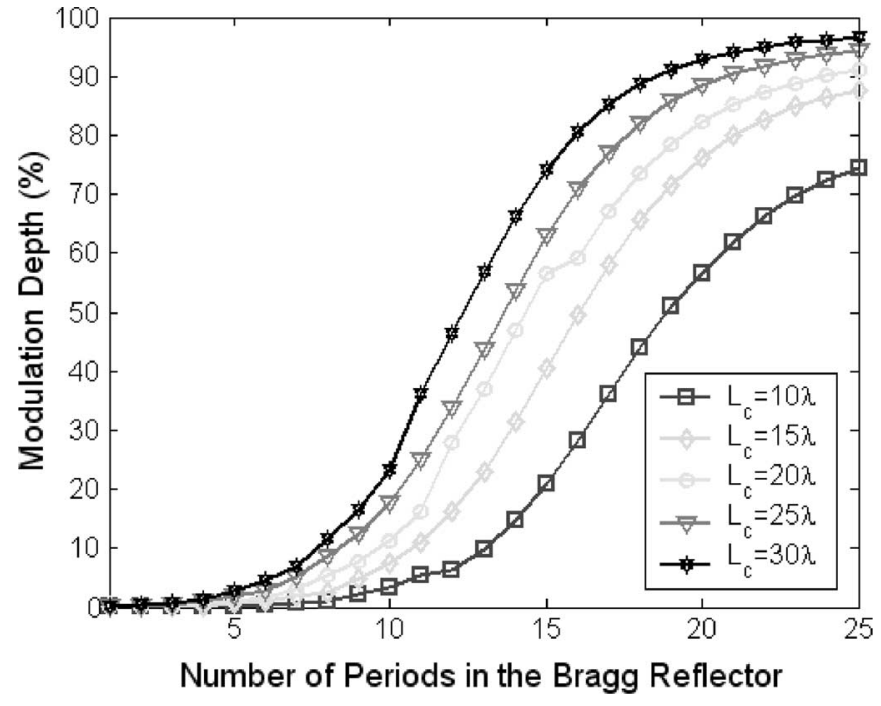

Fig. 9. The modulation depth for various cavity lengths with $100-\mathrm{nm}$ holes in the Bragg reflector. The maximum achievable modulation depth increases as the cavity length is increased, but this of course also increases the overall device length.

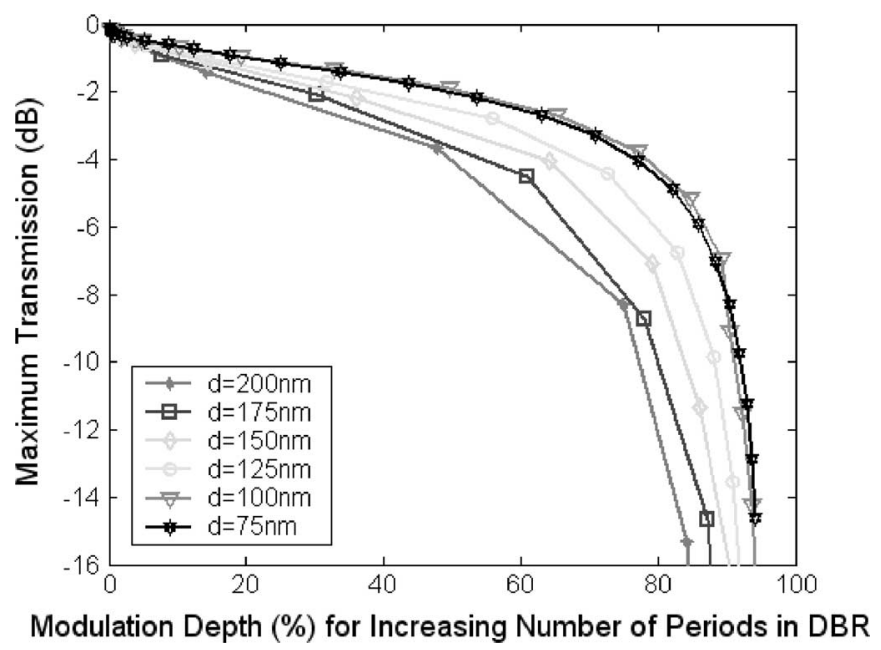

Fig. 10. There is a design tradeoff between the maximum transmission and the modulation depth for a given cavity length, shown here for a $25 \lambda$ cavity.

The design tradeoffs discussed above should be carefully considered once the requirements for a given application are known. Smaller holes offer better throughput, but at an increased cost of fabrication and longer device length, as a larger number of periods is necessary to achieve a narrow FWHM and large modulation depth. A longer cavity length decreases the FWHM and increases the modulation depth for a given hole size, which are both desirable effects, but it also increases the overall device length. An alternative to increasing the cavity length, if size is the concern for a particular application, is to increase the voltage applied across the EO polymer. This causes a greater change in the propagation constant in the cavity region and therefore increases the shift in the resonant wavelength, which in turn increases the modulation depth, as shown in Fig. 11.

Because the modulation depth can be improved by increasing the applied voltage, in addition to increasing the cavity length, 


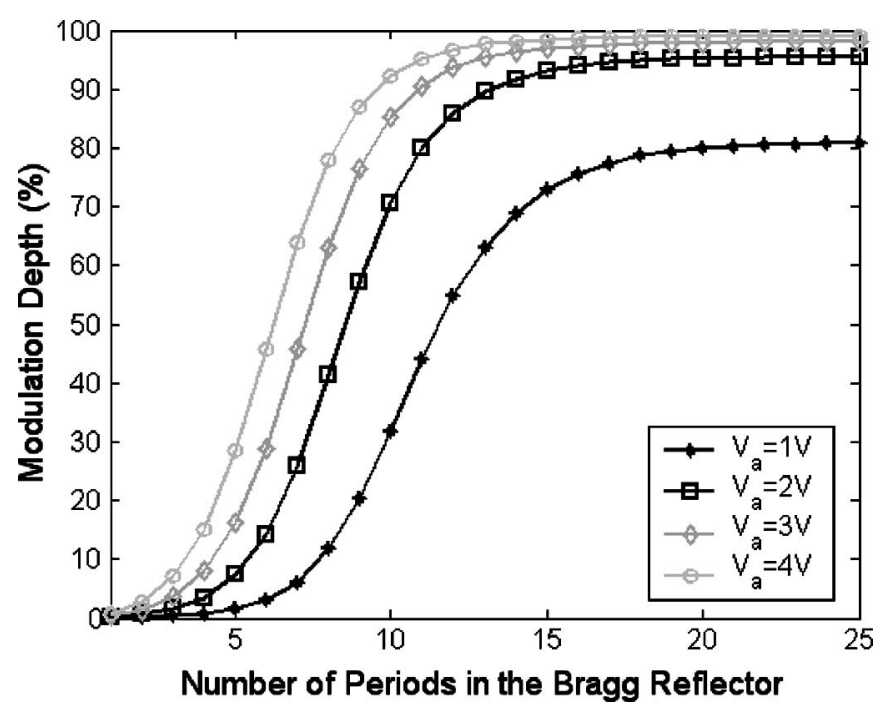

Fig. 11. The modulation depth can be increased by increasing the applied voltage rather than lengthening the cavity region.

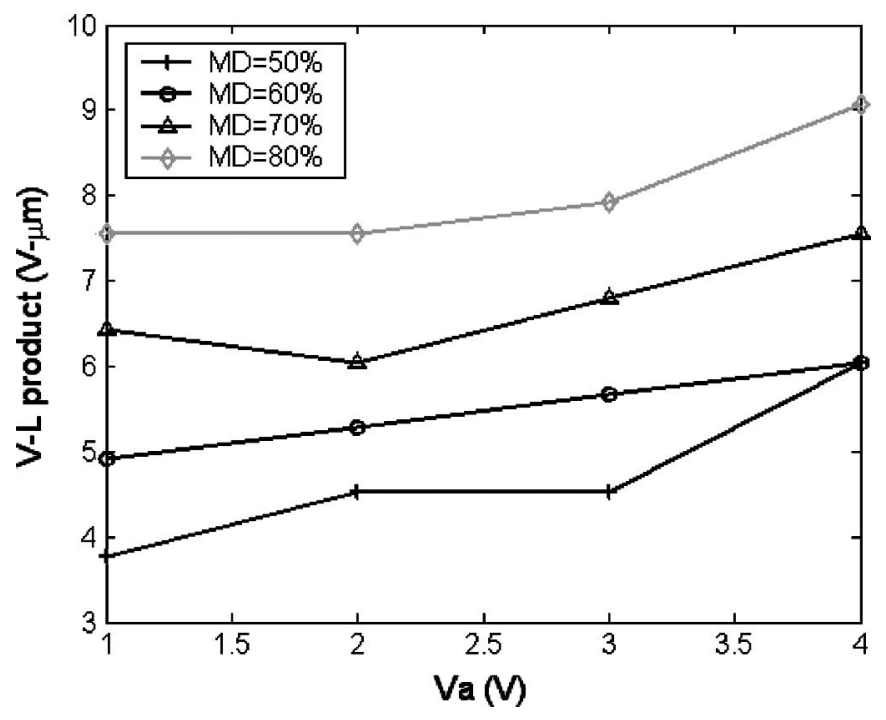

Fig. 12. The $V L$ product to achieve a given modulation depth. Increasing the applied voltage increases the $V L$ product somewhat, thereby showing that increasing the cavity length is slightly more beneficial than is increasing the applied voltage.

it is useful to look at the $V L$ product for a given modulation depth to see which property, larger applied voltage, or increased cavity length, will have the greatest effect on the response of the device. There is a significant decrease in the $V L$ products of this resonant cavity structure as compared to those of the MZ modulators, which are a measure of the voltage necessary to cause a phase change of $\pi$ for a given active arm length. While this definition does not strictly apply to the resonant cavity modulators, one can consider the voltage and cavity length necessary to achieve a given modulation depth. This provides a measure of the strength of the response of both the parameters, and is a useful comparison for Fabry-Perot-type modulators. This relationship is shown in Fig. 12, where the $V L$ product necessary to achieve a given modulation depth is plotted as a function of applied voltage. The upward slope of the $V L$ prod-

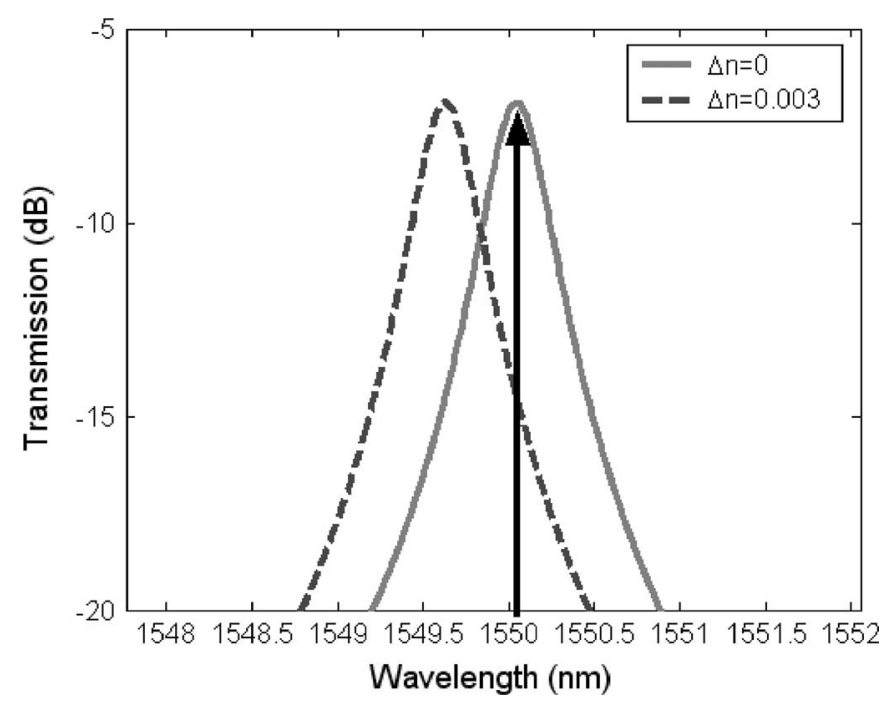

Fig. 13. Transmission spectrum illustrating the modulation depth of a device with 12 periods in the DBR, 100-nm hole diameters, and a $20 \lambda$ cavity region.

uct shows that it is slightly more beneficial to increase the cavity length, as opposed to the applied voltage. However, the slope is relatively low, and the final determination should depend more on application requirements, such as size or power constraints.

To illustrate the modulation around a given wavelength, a simulated transmission spectrum through a device with $12 \mathrm{Bragg}$ reflector periods and 100-nm circular holes on each side of a $20 \lambda(16 \mu \mathrm{m})$ cavity is shown in Fig. 13. The total device length for this modulator is only $31.6 \mu \mathrm{m}$, compared to an MZ structure with the hybrid waveguide, which would need an active length of $550 \mu \mathrm{m}$ for full modulation. The FWHM of this configuration is $0.38 \mathrm{~nm}$ and a modulation depth of $80.3 \%$ can be achieved with a refractive index change of only -0.003 , which, according to (2), corresponds to an applied voltage of $2.2 \mathrm{~V}$ with the silicon ridges in the cavity region serving as electrodes.

Another important consideration for this device is the achievable switching speed. There are three main factors that may limit the switching speed. First is the inherent response time of the polymer, which is expected to be on the order of femtoseconds [9]. Measurements on the EO polymer devices have been limited by external factors for modulation frequencies up to $20-100 \mathrm{GHz}$ [3], [9]. Because this is a resonant cavity device, the photon residence time may affect the switching speed unlike a traveling wave device, such as an MZ modulator. For the proposed design, we estimate the lifetime of photons in the resonant cavity to be less than $0.8 \mathrm{ps}$ [7]. Thus, the electrodes are expected to be the limiting factor in determining the switching speed of the device.

There are numerous resistances along the electrodes that will contribute to the total resistance, including contact resistance, bulk resistances, and spreading resistance between thin regions and taller silicon waveguide regions, as shown in Fig. 14. The resistance of the silicon ridge and the thin connecting region both depend on the height, width, and doping concentration. The doping concentration in the thin region should be very high in order to reduce the resistivity. However, high doping concentrations in the hybrid waveguide of the resonant cavity 


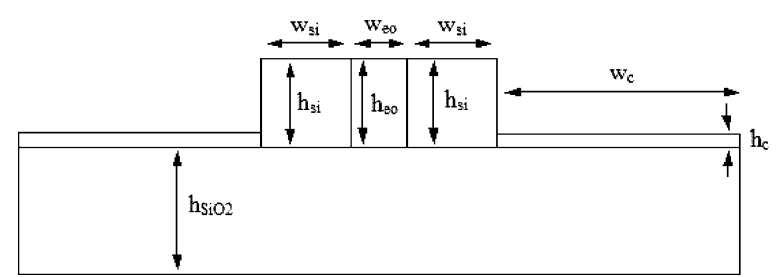

Fig. 14. Cross section of the hybrid waveguide including the heavily doped connecting regions of height $h_{c}$.

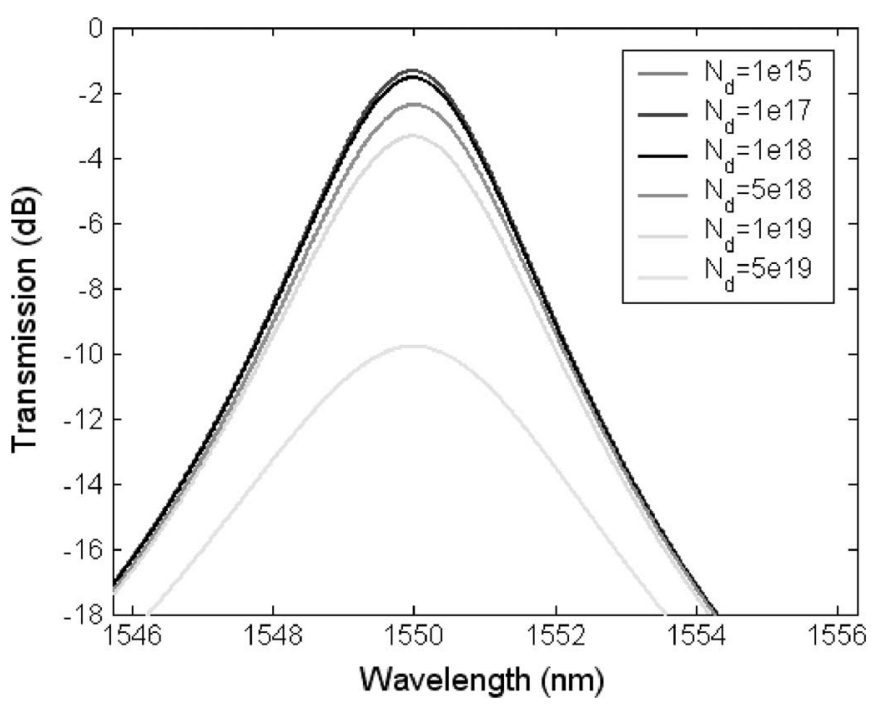

Fig. 15. Transmission spectrum for a $20 \lambda$ cavity illustrating the increased loss due to the doping in the silicon ridges.

region can significantly increase the attenuation of an optical wave as the attenuation coefficient increases by approximately $1 \times 10^{-18} N_{\mathrm{d}}\left(\mathrm{cm}^{-3}\right)$, where $N_{\mathrm{d}}$ is the doping concentration [11]. Therefore, the silicon ridges, which support a portion of the wave in the hybrid structure (as shown in Fig. 4), must be more lightly doped than the thin connecting regions. The increased transmission loss is illustrated in Fig. 15 and shows that the doping concentration in the silicon ridges should not exceed $10^{18} \mathrm{~cm}^{-3}$ in order to have a negligible change in the transmission at the resonant wavelength. Including the resistances and capacitances of the structure, the resulting time constant for the structure with $250 \mathrm{~nm}$ square silicon ridges, a $100 \mathrm{~nm}$ EO polymer core, and a $20 \mathrm{~nm}$ high connecting silicon region is $\sim 2.25 \mathrm{ps}$, corresponding to a response speed in excess of $100 \mathrm{GHz}$, which will allow for the fast switching speeds necessary for integrated optics applications.

\section{CONCLUSION}

This resonant cavity modulator design results in a large modulation depth and narrow FWHM for a very short overall device length. The complex structure of this device requires 3-D simulation for accurate analysis, which is computationally expensive. However, recognizing the periodicity of the device structure and using cascade matrixes reduces the required simulation times dramatically and provides an approach that could be used for optimization of many integrated optics devices. Incorporation of the hybrid waveguide structure in the resonant cavity region requires that only a small voltage be applied to cause a significant change in the propagation constant and therefore shift the resonant wavelength of the device. The hybrid waveguide design is also beneficial because the EO polymer is not introduced until after the majority of the processing is completed, and therefore allows for the use of well-established CMOS processing, which should ease the transition to hybrid silicon/organic polymer devices.

\section{REFERENCES}

[1] A. Liu, R. Jones, L. Liao, D. Samara Rubio, D. Rubin, O. Cohen, R. Nicolaescu, and M. Paniccla, "A high-speed silicon optical modulator based on a metal-oxide-semiconductor capacitor," Nature, vol. 427, pp. 615-618, Feb. 2004.

[2] J. Luo, S. Liu, M. Haller, J. Kang, T. Kim, S. Jang, B. Chen, N. Tucker, H. Li, H. Tang, L. R. Dalton, Y. Liao, B. H. Robinson, and A. K. Jen, "Recent progress in developing highly efficient and thermally stable nonlinear optical polymers for electro-optics," Proc. SPIE, vol. 5351, pp. 36-43, 2004.

[3] Y. Shi, C. Zhang, H. Zhang, J. H. Bechtel, L. R. Dalton, B. H. Robinson, and W. H. Steier, "Low (sub-1-volt) halfwave voltage polymeric electrooptic modulators achieved by controlling chromophore shape," Science, vol. 288, pp. 119-122, Apr. 2000.

[4] R. C. Twu, C. Y. Chang, and W. S. Wang, "A Zn-diffused Mach-Zehnder modulator on lithium niobate at $1.55 \mu \mathrm{m}$ wavelength," Microw. Opt. Technol. Lett., vol. 43, pp. 142-144, Mar. 2004.

[5] C. A. Barrios, V. R. Almeida, and M. Lipson, "Low-power-consumption short-length and high modulation-depth silicon electrooptic modulator," J. Lightw. Technol., vol. 21, pp. 1089-1098, Apr. 2003.

[6] C. A. Barrios, V. R. Almeida, R. R. Panepucci, B. S. Schmidt, and M. Lipson, "Compact silicon tunable Fabry-Perot resonator with low power consumption," IEEE Photon. Technol.Lett., vol. 16, no. 2, pp. 506508, Feb. 2004.

[7] P. Rabiei, W. H. Steier, C. Zhang, and L. R. Dalton, "Polymer micro-ring filters and modulators," J. Lightw. Technol., vol. 20, no. 11, pp. 19681975, Nov. 2002.

[8] V. R. Almeida, Q. Xu, C. A. Barrios, and M. Lipson, "Guiding and confining light in void nanostructures," Opt. Lett., vol. 29, pp. 1209-1211, Jun. 2004.

[9] L. Dalton et al., "Organic electro-optic materials," Proc. SPIE, vol. 5621, pp. 93-104, 2004.

[10] D. M. Kearns and R. W. Beatty, Basic Theory of Waveguide Junctions and Introductory Microwave Network Analysis. Oxford, U.K.: Pergamon, 1967.

[11] R. G. Hunsperger, Integrated Optics Theory and Technology. Berlin, Germany: Springer-Verlag, 2002.

Kjersti K. McLauchlan (S'00) received the B.S. degree in electrical engineering from Embry-Riddle Aeronautical University, Prescott, AZ, in 2003, and the M.S. degree in electrical engineering from the University of Washington, Seattle, in 2005. She is currently working toward the Ph.D. degree in electrical engineering at the University of Washington, Seattle.

Her current research interests include integrated optics and photonics device modeling.

Scott T. Dunham (M'94-SM'00) received the B.S. degree from Cornell University, Ithaca, NY, in 1979 and the M.S. and Ph.D. degrees from Stanford University, Stanford, CA, in 1980 and 1985, respectively, all in electrical engineering.

In 1980 and 1981, he was a Member of the Technical Staff, Bell Telephone Laboratories. In 1985, he joined the faculty of the Electrical and Computer Engineering Department, Boston University. In 1999, he joined the University of Washington, Seattle, WA, where he is currently a Professor of electrical engineering, as well as an Adjunct Professor of physics and Adjunct Professor of materials science and engineering. His current research interests include nanotechnology modeling, using a wide range of approaches including density functional theory, kinetic lattice Monte Carlo, and continuum reaction-diffusion equations. 